\title{
Distance Between External Cortical Bone and Mandibular Canal for Harvesting Ramus Graft: A Human Cadaver Study
}

Daylene Jack-Min Leong, * Jingjing Li, ${ }^{\dagger}$ Ildefonso Moreno, * and Hom-Lay Wang*

Background: The aims of this study are to determine the distance of the external surface of the buccal cortical plate to the inferior alveolar canal in the mandibular molar region and to propose a safe thickness for harvesting a mandibular ramus block graft.

Methods: Thirty-four cadavers consisting of 26 dentate jaws and eight jaws in the edentulous molar region were used in this study. All mandibular ramus grafts were harvested by one investigator with the grafts extending from the external oblique ridge (EOR) and $15 \mathrm{~mm}$ inferiorly in the apico-coronal direction and extending from the mid-buccal aspect of the first molar to the mid-buccal aspect of the third molar in the mesiodistal direction. Measurements were made of the cortical bone thickness of the harvested ramus graft and from the cementoenamel junction (CEJ) to the EOR, the CEJ to the mandibular border, and the CEJ to the inferior alveolar nerve (IAN).

Results: The average buccal cortical plate thickness in dentate mandibles was $2.76 \pm 0.13 \mathrm{~mm}$, whereas in edentulous posterior mandibles it was $2.52 \pm 0.32 \mathrm{~mm}$. The IANs were exposed in all jaws but were intact.

Conclusion: The safe thickness to harvest ramus grafts was determined to be 2.5 to $3.0 \mathrm{~mm}$. J Periodontol 2010;81:239243.

\section{KEY WORDS}

Alveolar ridge augmentation; edentulous jaw; inferior alveolar nerve.

\footnotetext{
* Department of Periodontics and Oral Medicine, School of Dentistry, University of Michigan, Ann Arbor, MI.

$\dagger$ Currently, private practice, Los Angeles, CA; formerly, Department of Periodontics and Oral Medicine, School of Dentistry, University of Michigan.
}

$\longrightarrow$ everal types of bone grafts are available for use in alveolar ridgeaugmentation procedures. These include autogenous bone grafts that are obtained directly from the patient, allograft materials obtained from genetically dissimilar members of the same species consisting of frozen cancellous bone and marrow and freeze-dried bone, xenografts procured from different species, and alloplasts, which are synthetic or inorganic graft materials., ${ }^{1,2}$ With the advent and rapid development of allografts, several limitations of using autografts, such as morbidity, time spent on harvesting the graft, and the limited volume and dimension of bone available, were addressed. ${ }^{3}$ However, numerous studies ${ }^{4-8}$ using autogenous bone grafts in ridge-augmentation procedures consistently yielded favorable results, and autogenous bone grafts are still considered the "gold standard" in alveolar ridge augmentation. ${ }^{9,10}$ The choice of a secondary surgical site is often based on the quantity of desired bone and the type of desired graft. The ramus graft usually yields a graft that is longer in length but not as thick as a symphysis graft because of the proximity of the inferior alveolar canal to the buccal surface of the external oblique ridge (EOR). Currently, there is a paucity of literature providing data to aid our understanding of applied anatomy in this area of the oral cavity. Therefore, the aim of this study is to determine the

doi: 10.1902/jop.2009.090417 
distance of the external surface of the buccal plate to the inferior alveolar canal in the mandibular molar region and to propose a safe thickness for harvesting a mandibular ramus block graft to minimize the complications associated with this procedure.

\section{MATERIALS AND METHODS}

A total of 34 white cadavers consisting of 26 dentate jaws and eight edentulous jaws in the molar region were used in this study. A full-thickness buccal periosteal flap was reflected, and mandibular ramus grafts were harvested by two investigators (JL, DL), from the molar regions with the superior cut made along the EOR, the inferior cut $15 \mathrm{~mm}$ from the superior cut, and the anterior and posterior cuts made on the mid-buccal aspect of the first and third molars (Fig. 1 ). These cuts were performed using a microsaw, and a bone chisel was used to engage the superior cut. The ramus graft was procured by gently malleting the entire length of the cut along the EOR. Care was taken to place the chisel parallel to the lateral surface of the ramus to avoid injury to the inferior alveolar nerve (IAN). In all 34 cadavers, a rectangular piece of graft was obtained with the exposure of the IAN, but the nerve was kept intact in all jaws.

Four different measurements were made on the buccal aspect of the molars by a calibrated examiner (DL): 1) the distance from the cemento-enamel junction to the EOR (CEJ-EOR), 2) the distance from the CEJ to the mandibular border (CEJ-MandB), 3) the distance from the CEJ to the IAN (CEJ-IAN), and 4) the cortical bone thickness of the harvested block graft. In edentulous jaws, the CEJ-EOR distance was measured from the mid-crestal ridge position to the EOR. Whenever a molar tooth was missing, measurements were taken $15 \mathrm{~mm}$ away from the distal surface of the terminal tooth. In edentulous jaws, each molar measurement was taken $15 \mathrm{~mm}$ apart from the distal surface of the second premolar. All measurements were made with a single Boley gauge caliper. With the data collected, $Q-Q$ plots were charted, which indicated that the data were normally distributed. The data collected were subsequently statistically analyzed using the independent two-sample $t$ test.

\section{RESULTS}

All measured distances were averaged as a combined group according to dentate and edentulous groups. On average, CEJ-EOR values were greater for dentate jaws compared to the crestal ridge-to-EOR distance in edentulous jaws $(8.76 \pm 1.62 \mathrm{~mm}$ versus $6.18 \pm 1.47 \mathrm{~mm}$, respectively), although the difference was not statistically significantly different $(P=$ 0.110). Correspondingly, CEJ-MandB (22.27 \pm $1.63 \mathrm{~mm})$ and CEJ-IAN (12.24 $\pm 0.58 \mathrm{~mm})$ values were greater in dentate jaws compared to the crestal ridge-to-MandB (17.45 $\pm 1.22 \mathrm{~mm})$ and crestal ridgeto-IAN $(9.98 \pm 0.70 \mathrm{~mm})$ distances in edentulous jaws, and both of these differences were statistically significantly different $(P<0.05$ for both) (Table 1$)$.

On the other hand, the difference between the buccal cortical plate thickness from dentate jaws was not statistically significantly larger compared to that from edentulous jaws, although a slightly thicker graft was obtained in the former. The average buccal cortical plate thickness in dentate mandibles was $2.76 \pm$ $0.30 \mathrm{~mm}$, whereas that in edentulous posterior mandibles was $2.52 \pm 0.32 \mathrm{~mm}(P=0.309)$. The mean buccal cortical plate thickness for all 34 jaws was $2.7 \pm 0.15 \mathrm{~mm}$. The $95 \%$ confidence interval obtained for this mean cortical bone thickness was 2.436 to 2.9636 .
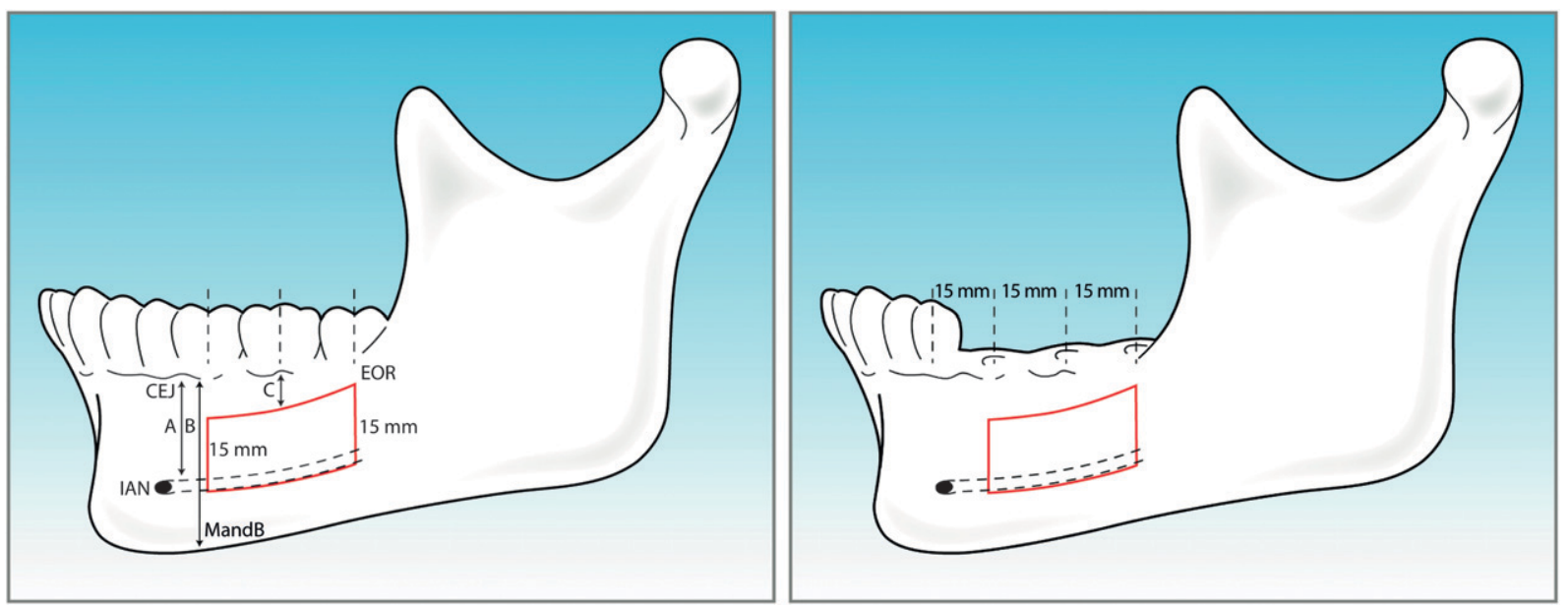

Figure I.

Diagrams illustrating the location of ramus-graft harvesting and locations of each measurement for dentate jaws (left) and edentulous sites (right). $A=C E J-I A N ; B=C E J-M a n d B ; C=C E J-E O R$. 
Table I.

\section{Measurements (mm; mean \pm SD) in Each Tooth Location}

\begin{tabular}{|c|c|c|c|c|c|}
\hline Measurement & First Molar & Second Molar & Third Molar & Mean \pm SD & $P$ Value \\
\hline \multicolumn{6}{|l|}{ CEJ-EOR } \\
\hline Dentate & $10.22 \pm 5.27$ & $9.05 \pm 3.94$ & $7.02 \pm 2.55$ & $8.76 \pm 1.62$ & \multirow[t]{3}{*}{0.110} \\
\hline Edentulous & $7.80 \pm 3.15$ & $5.80 \pm 2.42$ & $4.94 \pm 1.98$ & $6.18 \pm 1.47$ & \\
\hline Overall & $9.58 \pm 4.86$ & $8.28 \pm 3.87$ & $6.58 \pm 2.59$ & $8.15 \pm 1.50$ & \\
\hline \multicolumn{6}{|l|}{ CEJ-MandB } \\
\hline Dentate & $21.30 \pm 6.70$ & $21.37 \pm 5.61$ & $24.15 \pm 4.64$ & $22.27 \pm 1.63$ & \multirow[t]{3}{*}{$0.015 *$} \\
\hline Edentulous & $16.53 \pm 1.97$ & $16.99 \pm 2.27$ & $18.83 \pm 7.19$ & $17.45 \pm 1.22$ & \\
\hline Overall & $20.17 \pm 6.25$ & $20.34 \pm 5.34$ & $22.90 \pm 5.70$ & $21.14 \pm 1.53$ & \\
\hline \multicolumn{6}{|l|}{ CEJ-IAN } \\
\hline Dentate & $\mid 2.82 \pm 6.31$ & $11.67 \pm 5.58$ & $12.24 \pm 4.20$ & $12.24 \pm 0.58$ & \multirow[t]{3}{*}{$0.012 *$} \\
\hline Edentulous & $9.56 \pm 3.64$ & $9.58 \pm 3.73$ & $10.79 \pm 2.89$ & $9.98 \pm 0.70$ & \\
\hline Overall & $|2.05 \pm 5.9|$ & $11.18 \pm 5.23$ & $11.90 \pm 3.94$ & $\mid 1.71 \pm 0.47$ & \\
\hline \multicolumn{6}{|c|}{ Cortical bone thickness } \\
\hline Dentate & $2.87 \pm 0.54$ & $2.79 \pm 0.64$ & $2.61 \pm 0.71$ & $2.76 \pm 0.30$ & \multirow[t]{3}{*}{0.309} \\
\hline Edentulous & $2.43 \pm 0.75$ & $2.88 \pm 0.80$ & $2.26 \pm 0.36$ & $2.52 \pm 0.32$ & \\
\hline Overall & $2.76 \pm 0.62$ & $2.81 \pm 0.67$ & $2.53 \pm 0.65$ & $2.70 \pm 0.15$ & \\
\hline
\end{tabular}

* Significant at $P<0.05$.

\section{DISCUSSION}

The advantages of using mandibular bone grafts include excellent graft incorporation with little volume loss ${ }^{11-14}$ and reduction of surgical and anesthesia times because the donor and recipient sites are both in the same operating region. ${ }^{11,14-16}$ Furthermore, autogenous grafts are associated with less morbidity from graft harvesting compared to those from extraoral sites. ${ }^{11,15,17,18}$ However, the harvesting procedure for mandibular ramus grafts are not without complications, including common complications such as pain, swelling, postoperative bleeding, mandibular fracture, and IAN damage, which is the most frequently reported complication. ${ }^{19,20}$ Furthermore, neurosensory disturbances in the lower lip or mental nerve area were found to occur even after $>1$ year after bilateral, sagittal, split-ramus surgeries, especially if the mandibular canal was in contact with the external cortical bone. ${ }^{21}$ Therefore, the present study was conducted to add to the little knowledge we have of the thickness of the buccal cortical plate in relation to the IAN to aid the surgeon in reducing neurologic injury when harvesting a ramus graft.

Generally, the cortical bone thickness in the mandibular retromolar area is $\sim 3.0$ to $3.5 \mathrm{~mm} .^{22}$ In a cadaver study by Rajchel et al., ${ }^{23}$ the thickness of the buccal cortical plate at the superior-inferior level of the mandibular canal was found to be thickest at the second molar region $(2.3 \pm 0.7 \mathrm{~mm})$. This was slightly different than found in the present study, as the buccal cortical plate thickness was found to be greatest at the first molar region $(2.9 \mathrm{~mm})$. Also, the mean thickness of the buccal cortical plate was quantified to be $\sim 1.92$ $\mathrm{mm}$ in the study by Rajchel et al., ${ }^{23}$ which compares to a thickness of $\sim 2.8 \mathrm{~mm}$ calculated in the present study for dentate jaws and $\sim 2.5 \mathrm{~mm}$ for edentulous jaws. This difference may be accounted for by the width of the marrow space. In the present study, a marrow space was not easily found due to the nature of cadaver specimens. For those that had a marrow space, the cancellous bone was not preserved during the harvesting procedure, and therefore, it was impossible to measure a distinct marrow space. Nonetheless, based on a previous study by Katranji et al., ${ }^{24}$ the width of the buccal cortical plate in the molar region of the dentate mandible was $1.98 \pm 0.81 \mathrm{~mm}$, whereas in the edentulous mandible it was $2.06 \pm$ $0.69 \mathrm{~mm}$. Hence, by simple subtraction, the width of the marrow space can be estimated to be $\sim 0.78 \mathrm{~mm}$ in the dentate posterior mandible and $\sim 0.46 \mathrm{~mm}$ in the edentulous posterior mandible.

As the IAN was exposed in all 34 cases with no damage to the nerve after procurement of the ramus graft, it is reasonable to infer that a safe thickness to harvest for a ramus graft in the region of the molars would be $\sim 2.5$ to $3.0 \mathrm{~mm}$. Moreover, the confidence interval calculated in this study is less than the conventional distance of $\sim 3.0$ to $3.5 \mathrm{~mm} .^{22}$ Therefore, there is significant evidence that the safety zone for harvesting a mandibular ramus graft is a depth of $\sim 2.5$ to $3.0 \mathrm{~mm}$ from the external surface of the buccal plate in the mandibular molar region. 
As mentioned, in dentate cadaver jaws, the CEJ is further away from the EOR and the IAN compared to the crestal ridge distance to the EOR and IAN in edentulous posterior mandibles. This observation could be explained by the fact that, with the loss of teeth, the alveolar ridge resorbs, ${ }^{25-27}$ therefore bringing the CEJ or the crestal ridge closer to the EOR and the IAN. In the present study, the crestal ridge-to-IAN distance (i.e., the alveolar ridge height to the IAN) was, on average, $9.98 \mathrm{~mm}$ in edentulous posterior mandibles. This adds information for the placement of implants in edentulous posterior mandibles, as it serves to caution the surgeon that $10-\mathrm{mm}$ implants are not always feasible in that region of the oral cavity. Short implants may have to be used in the posterior mandible to avoid injury to the IAN.

Although we minimized the variables as much as possible, there are still some limitations in the study. Some of these include a small sample size and the inability to absolutely control variables such as the age and gender of the cadavers obtained. These data, as well as the duration of edentulism in edentulous individuals, were not available at the time of the study.

\section{CONCLUSIONS}

Within the limits of this cadaver study, the mean cortical bone thickness of the ramus graft harvested from the mandibular molar region was $\sim 2.8 \mathrm{~mm}$ in dentate jaws and $\sim 2.5 \mathrm{~mm}$ in edentulous jaws. The overall mean cortical bone thickness was $2.7 \mathrm{~mm}$, and statistical analysis revealed that there was significant evidence that a ramus graft should be harvested with a thickness $\leq 3.0 \mathrm{~mm}$. As the IAN was exposed in all cases but not damaged, the safe thickness to harvest ramus grafts, contrary to common belief, is 2.5 to 3.0 $\mathrm{mm}$. Furthermore, with the loss of teeth, the distances of the crestal ridge and the EOR to the IAN decreases. Therefore, the surgeon must bear in mind that there is an increased risk of nerve involvement when procuring a ramus graft in the apico-coronal direction.

\section{ACKNOWLEDGMENTS}

The authors thank Mr. Chris Jung, supporting staff, School of Dentistry, University of Michigan, for his expertise in drawing Figure 1. This article was partially supported by the University of Michigan Periodontal Graduate Student Research Fund. The authors report no conflicts of interest related to this study.

\section{REFERENCES}

1. Mellonig JT, Bowers GM. Regenerating bone in clinical periodontics. J Am Dent Assoc 1990;121:497-502.

2. Parikh SN. Bone graft substitutes: Past, present, future. J Postgrad Med 2002;48:142-148.

3. Peleg M. Using allogenic block grafts to augment the alveolar ridge. Dent Implantol Update 2004;15:8994.
4. Cordaro L, Amade DS, Cordaro M. Clinical results of alveolar ridge augmentation with mandibular block bone grafts in partially edentulous patients prior to implant placement. Clin Oral Implants Res 2002; 13:103-111.

5. D'Addona A, Nowzari H. Intramembranous autogenous osseous transplants in aesthetic treatment of alveolar atrophy. Periodontol 2000 2001;27:148-161.

6. Roccuzzo M, Ramieri G, Spada MC, Bianchi SD, Berrone S. Vertical alveolar ridge augmentation by means of a titanium mesh and autogenous bone grafts. Clin Oral Implants Res 2004;15:73-81.

7. Proussaefs P, Lozada J, Kleinman A, Rohrer MD. The use of ramus autogenous block grafts for vertical alveolar ridge augmentation and implant placement: A pilot study. Int J Oral Maxillofac Implants 2002;17: 238-248.

8. von Arx T, Wallkamm B, Hardt N. Localized ridge augmentation using a micro titanium mesh: A report on 27 implants followed from 1 to 3 years after functional loading. Clin Oral Implants Res 1998;9:123-130.

9. Triplett RG, Schow SR. Autologous bone grafts and endosseous implants: Complementary techniques. J Oral Maxillofac Surg 1996;54:486-494.

10. Lundgren S, Rasmusson L, Sjostrom M, Sennerby L. Simultaneous or delayed placement of titanium implants in free autogenous iliac bone grafts. Histological analysis of the bone graft-titanium interface in 10 consecutive patients. Int J Oral Maxillofac Surg 1999; 28:31-37.

11. Sindet-Pedersen S, Enemark H. Reconstruction of alveolar clefts with mandibular or iliac crest bone grafts: A comparative study. J Oral Maxillofac Surg 1990;48:554-558; discussion 559-560.

12. Jensen J, Sindet-Pedersen S, Oliver AJ. Varying treatment strategies for reconstruction of maxillary atrophy with implants: Results in 98 patients. J Oral Maxillofac Surg 1994;52:210-216; discussion 216218.

13. Williamson RA. Rehabilitation of the resorbed maxilla and mandible using autogenous bone grafts and osseointegrated implants. Int $J$ Oral Maxillofac Implants 1996;11:476-488.

14. Misch CM. Comparison of intraoral donor sites for onlay grafting prior to implant placement. Int $J$ Oral Maxillofac Implants 1997;12:767-776.

15. Misch CM. Ridge augmentation using mandibular ramus bone grafts for the placement of dental implants: Presentation of a technique. Pract Periodontics Aesthet Dent 1996;8:127-135; quiz 138.

16. Braun TW, Sotereanos GC. Autogenous regional bone grafting as an adjunct in orthognathic surgery. J Oral Maxillofac Surg 1984;42:43-48.

17. Misch CM, Misch CE. The repair of localized severe ridge defects for implant placement using mandibular bone grafts. Implant Dent 1995;4:261-267.

18. Jensen J, Sindet-Pedersen S. Autogenous mandibular bone grafts and osseointegrated implants for reconstruction of the severely atrophied maxilla: A preliminary report. J Oral Maxillofac Surg 1991;49: 1277-1287.

19. Nkenke E, Radespiel-Tröger M, Wiltfang J, SchultzeMosgau S, Winkler G, Neukam FW. Morbidity of harvesting of retromolar bone grafts: A prospective study. Clin Oral Implants Res 2002;13:514-521.

20. Clavero J, Lundgren S. Ramus or chin grafts for maxillary sinus inlay and local onlay augmentation: 
Comparison of donor site morbidity and complications. Clin Implant Dent Relat Res 2003;5:154-160.

21. Yamamoto R, Nakamura A, Ohno K, Michi K. Relationship of the mandibular canal to the lateral cortex of the mandibular ramus as a factor in the development of neurosensory disturbance after bilateral sagittal split osteotomy. J Oral Maxillofac Surg 2002;60: 490-495.

22. Smith BR, Rajchel JL, Waite DE, Read L. Mandibular anatomy as it relates to rigid fixation of the sagittal ramus split osteotomy. J Oral Maxillofac Surg 1991; 49:222-226.

23. Rajchel J, Ellis E 3rd, Fonseca RJ. The anatomical location of the mandibular canal: Its relationship to the sagittal ramus osteotomy. Int $J$ Adult Orthodon Orthognath Surg 1986;1:37-47.

24. Katranji A, Misch K, Wang HL. Cortical bone thickness in dentate and edentulous human cadavers. $J$ Periodontol 2007;78:874-878.
25. Carlsson GE, Thilander H, Hedegard B. Histologic changes in the upper alveolar process after extractions with or without insertion of an immediate full denture. Acta Odontol Scand 1967;25:21-43.

26. Tallgren A. The continuing reduction of the residual alveolar ridges in complete denture wearers: A mixedlongitudinal study covering 25 years. J Prosthet Dent 1972;27:120-132.

27. Abrams H, Kopczyk RA, Kaplan AL. Incidence of anterior ridge deformities in partially edentulous patients. J Prosthet Dent 1987;57:191-194.

Correspondence: Dr. Hom-Lay Wang, Department of Periodontics and Oral Medicine, School of Dentistry, University of Michigan, 1011 N. University Ave., Ann Arbor, MI 48109-1078. E-mail: homlay@umich.edu.

Submitted July 20, 2009; accepted for publication September 9, 2009. 